\title{
Anatomical and histomorphometric observations on the transfer of the anterior interosseous nerve to the deep branch of the ulnar nerve
}

The Journal of Hand Surgery

(European Volume)

2015, Vol. 40E(6) 591-596

(c) The Author(s) 2014

Reprints and permissions:

sagepub.co.uk/journalsPermissions.nav DOI: $10.1177 / 1753193414551909$

jhs.sagepub.com

@SAGE

\author{
T. L. Schenck ${ }^{1}$, J. Stewart ${ }^{2}$, S. Lin², M. Aichler ${ }^{3}$, \\ H.-G. Machens ${ }^{1}$ and R. E. Giunta ${ }^{2}$
}

\begin{abstract}
This study focuses on the anatomical and histomorphometric features of the transfer of the anterior interosseous nerve to the deep motor branch of the ulnar nerve. The transfer was carried out in 15 cadaver specimens and is described using relevant anatomical landmarks. Nerve samples of donor and target nerves were histomorphometrically analysed and compared. The superficial and the deep ulnar branches had to be separated from each other for a length of $67 \mathrm{~mm}$ (SD 12; range 50-85) to reach the site of coaptation. We identified a suitable site for coaptation lying proximal to the pronator quadratus muscle, $202 \mathrm{~mm}$ (SD 15; range 185-230) distal to the medial epicondyle of the humerus. The features of the anterior interosseous nerve included a smaller nerve diameter, smaller cross-sectional area of fascicles, fewer fascicles and axons, but a similar axon density. The histomorphometric inferiority of the anterior interosseous nerve raises a question about whether it should be transferred only to selected parts of the deep motor branch of the ulnar nerve.
\end{abstract}

Level III

Keywords

Ulnar nerve paresis, reinnervation, extraanatomic, axon count, peripheral nerve repair, microsurgery

\section{Introduction}

Major nerve injuries and the associated loss of sensory and motor functions severely affect the patient's quality of life. In the upper extremity, the ulnar nerve is the most commonly injured nerve (Kouyoumdjian, 2006). Recovery after ulnar nerve injuries is poorer with longer nerve defects and in more proximal injuries (Merle et al., 1986; Vastamäki et al., 1993). Because of the poor outcomes of proximal ulnar nerve repair, nerve transfers were introduced (Estrella and Mella, 2013; Harris, 1921; Jobe and Wright, 1998; Mackinnon and Novak, 1999). Nerve transfers involve the loss of an uninjured donor nerve, which is transferred and sutured to the distal stump of the injured target nerve (Mackinnon and Colbert, 2008). Nerve transfers are generally indicated in cases in which the time for regeneration through direct nerve repair is too long or recovery is impossible. Other reasons include a proximal nerve stump that is unavailable or inadequate and the avoidance of surgery in scarred tissue beds.
Wang and Zhu (1997) described the transfer of the anterior interosseous nerve (AIN) to the deep branch of the ulnar nerve (DBUN) to regain intrinsic hand muscle function. Battiston and Lanzetta (1999) described the reconstruction of the motor and sensory parts of the ulnar nerve. This study presents anatomical data on this nerve transfer

\footnotetext{
1Department of Plastic Surgery and Hand Surgery, University Hospital rechts der Isar, Technical University Munich, Germany ${ }^{2}$ Hand Surgery, Plastic Surgery and Aesthetic Surgery, Ludwig Maximilians University Munich, Germany ${ }^{3}$ Research Unit Analytical Pathology, Helmholtzzentrum München, German Research Center for Environmental Health, Munich, Germany
}

\section{Corresponding author:}

T. L. Schenck, Department of Plastic Surgery and Hand Surgery, University Hospital rechts der Isar, Technical University Munich, Ismaningerstr. 22, München, 81675, Germany.

Email: thilo.schenck@tum.de 


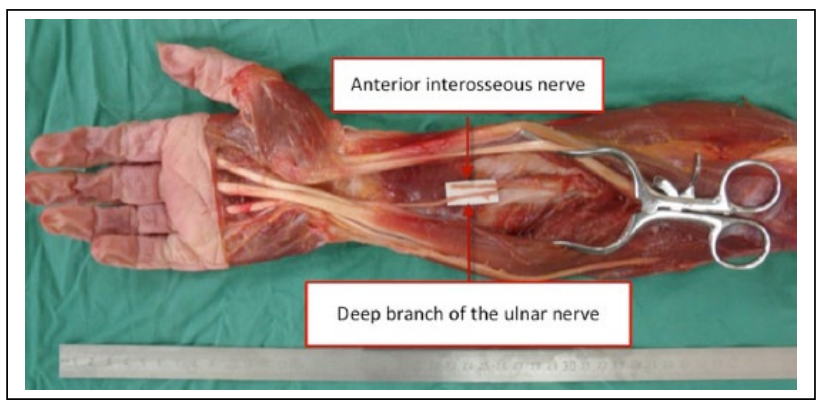

Figure 1. Transfer of the anterior interosseous nerve (AIN) to the dorsal branch of the ulnar nerve (DBUN).

and compares the histomorphometric characteristics of the two nerves.

\section{Methods}

\section{Anatomical dissection}

Anatomical measurements were made on 15 fresh cadaver upper limbs. The AIN was identified and transected proximal to the pronator quadratus (PQ) muscle. It was separated from the surrounding tissues in the proximal direction for a length of approximately $3-4 \mathrm{~cm}$ and transferred to the ulnar border of the $P Q$. The DBUN was identified within Guyon's canal and dissected from the fascicles of the superficial branch of the ulnar nerve to the level of the AIN. There, the nerves were coapted and the distances to relevant anatomical landmarks the medial epicondyle, the pisiform, and the dorsal cutaneous branch of the ulnar nerve (DCBUN) - were recorded (Figure 1).

\section{Histomorphometric analysis}

Nerve samples were excised from 14 fresh specimens at the level of the coaptation and fixed at $4^{\circ} \mathrm{C}$ in $2.5 \%$ glutaraldehyde with $0.1 \mathrm{M}$ sodium cacodylate buffer for $60 \mathrm{~min}$ (pH7.4; Science Services, Munich, Germany). After postfixation in $2 \%$ aqueous osmium tetraoxide, specimens were dehydrated in an ascending alcohol series $(30 \%-100 \%)$ and propylene oxide (Science Services, Munich, Germany). Samples were embedded in epoxy resin (Merck, Darmstadt, Germanyl preserving their orientation and cured for $24 \mathrm{~h}$ at $60^{\circ} \mathrm{C}$. Semithin transverse sections $(1 \mu \mathrm{m})$ were obtained with an ultramicrotome (Reichert Technologies, Munich, Germanyl, stained for $1 \mathrm{~min}$ with $1 \%$ toluidine blue (Sigma-Aldrich, Taufkirchen, Germanyl, and scanned at a 20x magnification with a Mirax Scannner (Carl Zeiss, Jena, Germany). The nerve diameter, fascicle number, and cross-sectional area of the individual fascicles were measured at 200x magnification (Figure 2(A) and (D)). Cross-sectional areas were measured using specialized software (Pannoramic Viewer 1.15; 3DHISTECH, Budapest, Hungary). The total areas of fascicles were calculated as the sum of the cross-sectional surfaces of all fascicles. Myelinated axons were counted semi-automatically at $600 \times$ magnification (Image J, version 1.42; $\mathrm{NIH}$, Bethesda, MD, USA) (Figure 2(B), (C), (E), and (F)). The low cut-off value for inclusion of axons was set at $4 \mu \mathrm{m}$. Axon density was calculated as the ratio of axon number and fascicle area. Donor-to-target ratios of the means were calculated for all parameters. Donor-to-target axon ratios were calculated for each specimen.

Statistical analysis was done using a two-tailed $t$-test with $p \leq 0.05$ being considered as significant. All data are given as the mean and standard deviation (SD), along with the range when appropriate.

\section{Results}

\section{Anatomical dissection}

The AIN and the DBUN were identified in all specimens without any anatomical variations. After dissection, the AIN could be transferred towards the proximal and ulnar border of the $P Q$ without loss of relevant length. The retrograde interfascicular neurolysis of the superficial and deep ulnar branch starting at their division at the pisiform never reached the takeoff of the DCBUN, which could be preserved in all cases. After neurolysis of the DBUN and nerve mobilization, a tension-free coaptation was possible at the proximal and ulnar border of the $P Q$ in every specimen. Results of the anatomical measurements for the location of the coaptation, the neurolysis distance, and the takeoff of the DCBUN are shown in Figure 3. Nerve diameters are shown in Figure 4(A).

\section{Histomorphometry}

The comparison of donor-to-target nerves revealed that the AIN had a significantly smaller diameter, smaller fascicular cross-sectional area, fewer fascicles and axons, and a smaller axon density. Histomorphometric results are given in Figure 4 and Table 1. Individual donor-to-target axon count ratios for each specimen are depicted in Figure 5.

\section{Discussion}

One major factor for success in treating peripheral nerve injuries is the length of time between axotomy and reinnervation (Brown et al., 2009a). Nerve transfers increase the chance for successful reinnervation by shortening both the regeneration time and distance. 


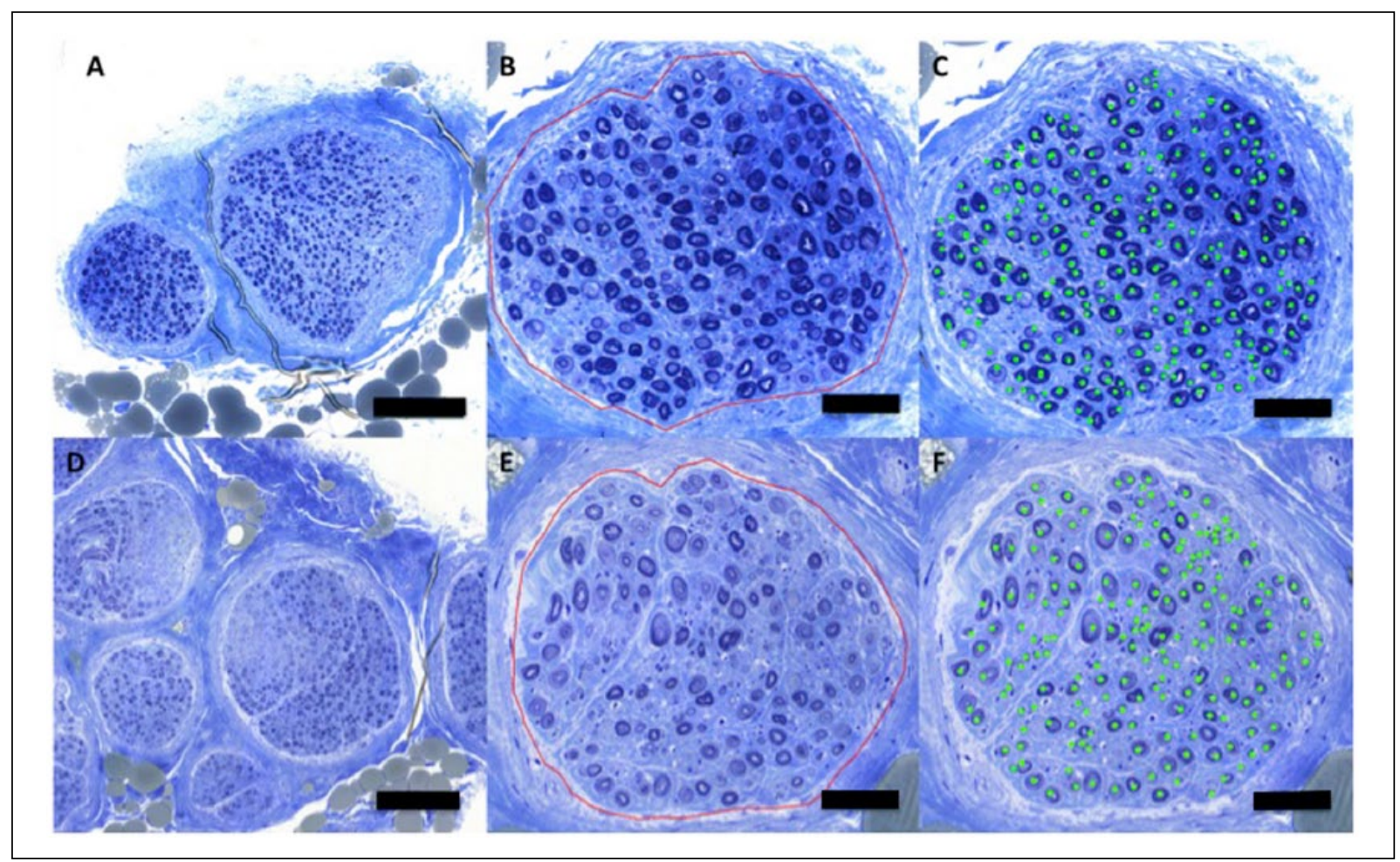

Figure 2. Semithin sections of the AIN (A), (B), (C) and the DBUN (D), (E), (F) from the coaptation site. Calibration bars represent $200 \mu \mathrm{m}(\mathrm{A})$ and (D) and $50 \mu \mathrm{m}(\mathrm{B}),(\mathrm{C}),(\mathrm{E})$, and (F).

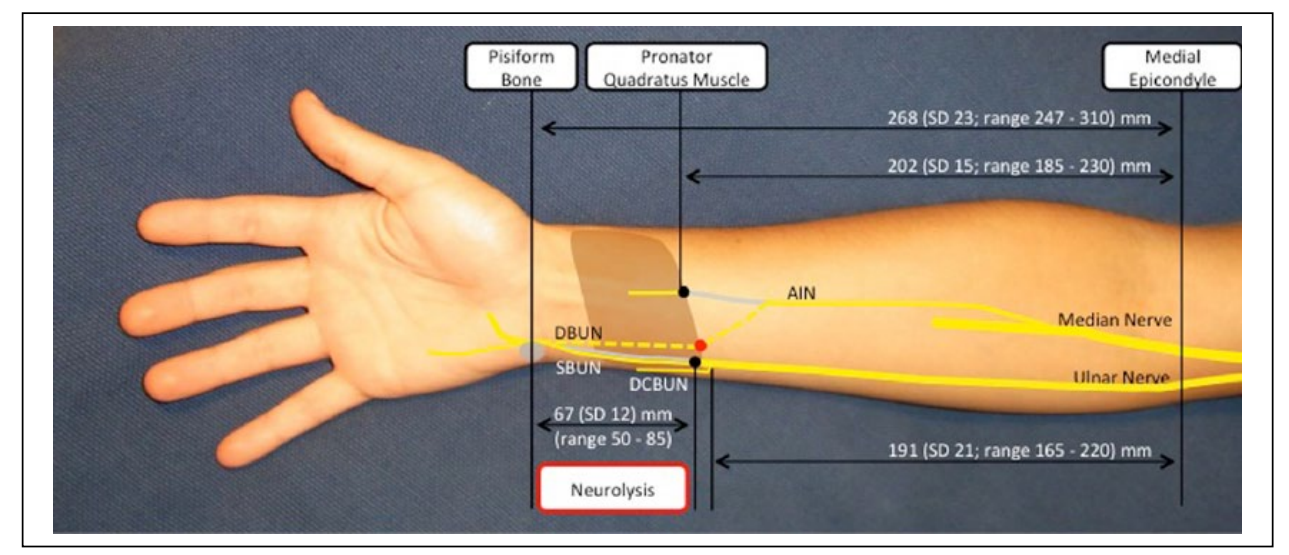

Figure 3. Black dots highlight the location of nerve dissection. The courses of the AIN and DBUN before their transposition are shown in grey. Interrupted lines illustrate their positions after the transfer. The location of the coaptation is depicted by the red dot. For reasons of clarity the median nerve is shown just to the level shortly beyond the takeoff of the AIN. The pronator quadratus muscle is highlighted in brown, the pisiform in grey.

AIN: anterior interosseous nerve; DBUN: deep branch of the ulnar nerve; DCBUN: dorsal cutaneous branch of the ulnar nerve; SBUN: superficial branch of the ulnar nerve.

In the treatment of facial paralysis and brachial plexus injuries, nerve transfers are well-established procedures (Battiston et al., 2009; Flores, 2013; Klebuc, 2011; Kozin, 2008; Terzis and Barmpitsioti, 2012). In all specimens, the AIN could be transferred to the proximal and ulnar border of the $P Q$ without loss of relevant length. Mobilization of the AIN did not interfere with its branches to the long flexors. The sensory DCBUN could be preserved in all cases (Figure 3). Loss of sensation could thus be avoided, keeping the nerve available as a donor for sensory transfers (Bedeschi et al., 1984; Brown et al., 2009b).

The proximity to target muscles is also a factor for success in nerve repair because denervated muscles 



Figure 4. Nerve diameters (A), fascicle numbers (B), total cross-sectional fascicle areas (C), axon numbers (D), and axon densities (E) were compared between the donor AIN (left column) and the target DBUN (right column). The AIN had significantly $(p<0.05)$ lower values in all parameters. All data are presented as mean and SD (shown by the whisker).

AIN: anterior interosseous nerve; DBUN: deep branch of the ulnar nerve.

Table 1. Donor-to-target (AIN: DBUN) ratios of histomorphometric nerve characteristics.

\begin{tabular}{ll}
\hline Parameter & AIN:DBUN \\
\hline Nerve diameter $(\mathrm{mm})$ & $1: 2.0$ \\
Fascicle number & $1: 3.7$ \\
Fascicle area $\left(\mathrm{mm}^{2}\right)$ & $1: 3.6$ \\
Axon number & $1: 4.8$ \\
Axon density (axons $\left./ \mathrm{mm}^{2}\right)$ & $1: 1.4$ \\
\hline
\end{tabular}

AIN, anterior interosseous nerve; DBUN, deep branch of ulnar nerve.

degrade over time (Viguie et al., 1997). The speed of nerve regeneration is usually given by rule of thumb as $1 \mathrm{~mm}$ of nerve regeneration per day. We found the coaptation point to lie $202 \mathrm{~mm}$ (SD 15; range 185-230) distal to the medial epicondyle (Figure 3 ). This allows an estimation of the gains in reinnervation time and distance by this nerve transfer. For ulnar nerve injuries at the elbow level, the reinnervation distance is cut by more than half and the reinnervation time gained can be estimated to be 6.5 months. Our measurements also allow an estimate of the time span from surgery until the regaining of muscle function. $A$ reinnervation time of about 100 days can be estimated, taking into account the distance from the point of coaptation to the target muscles. These time spans should be considered with regard to the limited window of time for nerve transfers. Our measurements are in line with other studies and equally valid for the end-to-side variant of this transfer (Barbour et al., 2012; Doyle and Botte, 2003; Robert et al., 2011; Tubbs et al., 2006). There is a variable number of interchanging nerve fibres between the DBUN and superficial branch of the ulnar nerve, which may be cut if they are small (Brown et al., 2009b). Our anatomical results present the AIN as a 


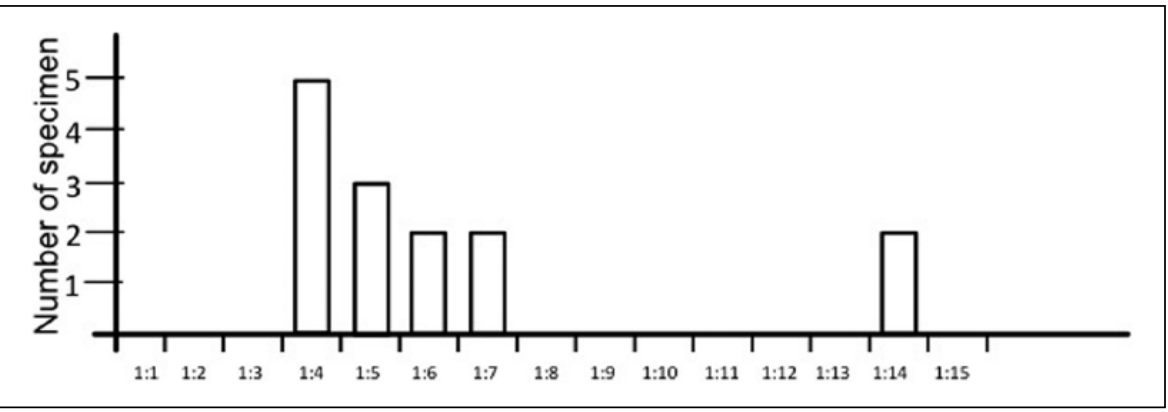

Figure 5. Individual donor-to-target axon count ratios. This figure depicts the frequency of the individual ratios.

Table 2. Comparison of donor-to-target axon ratios with previous reports.

\begin{tabular}{|c|c|c|c|c|}
\hline & $n$ & AIN:DBUN & $\begin{array}{l}\text { Location of sample collection } \\
\text { for the AIN }\end{array}$ & $\begin{array}{l}\text { Location of sample collection } \\
\text { for the DBUN }\end{array}$ \\
\hline Our data & 14 & $1: 4.8$ & $\begin{array}{l}\text { Proximal to pronator quadratus } \\
\text { (at the coaptation) }\end{array}$ & $\begin{array}{l}\text { Proximal to pronator } \\
\text { quadratus (at the coaptation) }\end{array}$ \\
\hline Wang and Zhu, (1997) & 7 & $1: 1.5$ & Proximal to pronator quadratus & Pisiform bone \\
\hline Üstün et al., (2001) & 10 & $1: 1.3$ & Proximal to pronator quadratus & Guyon's canal \\
\hline Brown et al., (2009b) & Not given & $1: 2$ & Not described & Not described \\
\hline Novak and Mackinnon, 2002. & Not given & $1: 4.1$ & Proximal to pronator quadratus & Not described \\
\hline
\end{tabular}

AIN: anterior interosseous nerve; DBUN: deep branch of ulnar nerve.

suitable donor for the DBUN and should be of help in planning this procedure.

Comparisons between donor and target nerves by their histomorphometric characteristics are commonly accepted methods for estimating the results of nerve transfers (Boutros et al., 1999; Rodriguez et al., 2011). Our comparison revealed the AIN to be significantly smaller in all histomorphometric aspects (Table 1). However, nerve transfers with a donor that is smaller than the target nerve can be successful. Axons in the proximal stump can undergo collateral sprouting that increases the number of axons by $3-4$ times (Jiang et al., 2007). Experiments with different donor-to-target axon ratios in rabbits have shown useful motor recovery beginning at a 1:3 ratio (Lutz et al., 2000). Based on these studies, the average axon ratio of 1:4.8 in the current study can be regarded as low. Although the absolute numbers of semi-automatic axon counts vary between studies owing to inclusion criteria, axon ratios are comparable (Raimondo et al., 2009). We calculated axon ratios for this nerve transfer from the axon numbers in other studies (Table 2). Interestingly, two studies used DBUN samples from the level of the pisiform (Üstün et al., 2001; Wang and Zhu, 1997). The two other studies did not state the location where the samples were collected (Brown et al., 2009b; Novak and Mackinnon, 2002). In contrast, we analysed the nerves directly at the site of coaptation.
The majority of specimens had donor-to-target axon ratios of $1: 4$ and 1:5, which can be considered to be close to the commonly accepted threshold of 1:3. Clinical reports of satisfactory outcomes could indicate that the poorer axon ratios are adequate for this particular nerve transfer (Battiston and Lanzetta, 1999; Brown et al., 2009b; Mackinnon and Novak, 1999; Wang and Zhu, 1997).

Two specimens presented with very poor donorto-target axon ratios of approximately 1:13 (Figure 5). Sporadically occurring poor axon ratios might explain cases with a poor clinical outcome (Wang and Zhu, 1997). The poor ratio could be addressed by transferring the AIN to selected DBUN fascicles that are expected to be most helpful for hand function in the individual patient.

\section{Acknowledgement}

The authors wish to acknowledge the substantial support of Professor Dr Helmut Gruber at the Institute for Anatomy, Medical University Vienna, Austria.

\section{Authors' note}

This article was presented at the annual FESSH Congress, 2013 in Antalya.

\section{Conflict of interests}

None declared. 


\section{Funding}

This research received no specific grant from any funding agency in the public, commercial, or not-for-profit sectors.

\section{References}

Barbour J, Yee A, Kahn LC, Mackinnon SE. Supercharged end-to-side anterior interosseous to ulnar motor nerve transfer for intrinsic musculature reinnervation. J Hand Surg Am. 2012, 37: 2150-9.

Battiston B, Lanzetta M. Reconstruction of high ulnar nerve lesions by distal double median to ulnar nerve transfer. J Hand Surg. 1999, 24A: 1185-91.

Battiston B, Artiaco S, Conforti LG, Vasario G, Tos P. Endto-side nerve suture in traumatic injuries of brachial plexus: review of the literature and personal case series. J Hand Surg Eur. 2009, 34: 656-9.

Bedeschi P, Celli L, Balli A. Transfer of sensory nerves in hand surgery. J Hand Surg Br. 1984, 9: 46-9.

Boutros S, Nath RK, Yüksel E, Weinfeld AB, Mackinnon SE. Transfer of flexor carpi ulnaris branch of the ulnar nerve to the pronator teres nerve: histomorphometric analysis. J Reconstr Microsurg. 1999, 15: 119-22.

Brown JM, Shah MN, Mackinnon SE. Distal nerve transfers: a biology-based rationale. Neurosurg Focus. 2009a, 26: E12.

Brown JM, Yee A, Mackinnon SE. Distal median to ulnar nerve transfers to restore ulnar motor and sensory function within the hand: technical nuances. Neurosurgery. 2009b, 65: 966-77.

Doyle JR, Botte MJ. Surgical anatomy of the hand and upper extremity. Philadelphia, Lippincott Williams \& Wilkins, 2003: 189, 193, 206, 209.

Estrella EP, Mella PM. Double nerve transfer for elbow flexion in obstetric brachial plexus injury: a case report. J Plast Reconstr Aesthet Surg. 2013, 66: 423-6.

Flores LP. Results of surgical techniques for re-innervation of the triceps as additional procedures for patients with upper root injuries. J Hand Surg Eur. 2013, 38: 248-56.

Harris RI. The treatment of irreparable nerve injuries. Can Med Assoc J. 1921, 11: 833-41.

Jiang BG, Yin XF, Zhang DY, Fu ZG, Zhang HB. Maximum number of collaterals developed by one axon during peripheral nerve regeneration and the influence of that number on reinnervation effects. Eur Neurol. 2007, 58 : $12-20$.

Jobe TM, Wright PE. Peripheral nerve injuries. In: Canale ST (Ed.) Campbell's operative orthopaedics. St. Louis, Mosby, 1998, Vol. 4: 3827-94.

Klebuc MJ. Facial reanimation using the masseter-tofacial nerve transfer. Plast Reconstr Surg. 2011, 127 : 1909-15.

Kouyoumdjian JA. Peripheral nerve injuries: a retrospective survey of 456 cases. Muscle Nerve. 2006, 34: 785-8.
Kozin SH. Nerve transfers in brachial plexus birth palsies: indications, techniques, and outcomes. Hand Clin. 2008, 24: 363-76.

Lutz BS, Chuang DC, Chuang SS, Hsu JC, Ma SF, Wei FC. Nerve transfer to the median nerve using parts of the ulnar and radial nerves in the rabbit - effects on motor recovery of the median nerve and donor nerve morbidity. J Hand Surg Br. 2000, 25: 329-35.

Mackinnon SE, Colbert SH. Nerve transfers in the hand and upper extremity surgery. Tech Hand Up Extrem Surg. 2008, 12: 20-33.

Mackinnon SE, Novak CB. Nerve transfers: new options for reconstruction following nerve injury. Hand Clin. 1999, 15: 643-66.

Merle M, Amend P, Cour C, Foucher G, Michon J. Microsurgical repair of peripheral nerve lesions. Periph Nerve Repair Regen. 1986, 2: 17-26.

Novak CB, Mackinnon SE. Distal anterior interosseous nerve transfer to the deep motor branch of the ulnar nerve for reconstruction of high ulnar nerve injuries. $J$ Reconstr Microsurg. 2002, 18: 459-64.

Raimondo S, Fornaro M, Di Scipio F, Ronchi G, GiacobiniRobecchi MG, Geuna S. Chapter 5: Methods and protocols in peripheral nerve regeneration experimental research: part II-morphological techniques. Int Rev Neurobiol. 2009, 87: 81-103.

Robert M, Blanc C, Gasnier P, Le Nen D, Hu W. Neurotization of the deep branch of ulnar nerve with anterior interosseous nerve: anatomic study. Chir Main. 2011, 30: 406-9.

Rodriguez A, Chuang DC, Chen KT, Chen RF, Lyu RK, Ko YS. Comparative study of single-, double-, and triplenerve transfer to a common target: experimental study of rat brachial plexus. Plast Reconstr Surg. 2011, 127 : 1155-62.

Terzis JK, Barmpitsioti A. Our experience with triceps nerve reconstruction in patients with brachial plexus injury. $J$ Plast Reconstr Aesthet Surg. 2012, 65: 590-600.

Tubbs RS, Custis JW, Salter EG, Wellons JC, Blount JP, Oakes WJ. Quantitation of and superficial surgical landmarks for the anterior interosseous nerve. J Neurosurg. 2006, 104: 787-91.

Üstün ME, Oğün TC, Büyükmumcu M, Salbacak A. Selective restoration of motor function in the ulnar nerve by transfer of the anterior interosseous nerve. An anatomical feasibility study. J Bone Joint Surg Am. 2001, 83: 549-52.

Vastamäki M, Kallio PK, Solonen KA. The results of secondary microsurgical repair of ulnar nerve injury. J Hand Surg Br. 1993, 18: 323-6.

Viguie CA, Lu DX, Huang SK, Rengen H, Carlson BM: Quantitative study of the effects of long-term denervation on the extensor digitorum longus muscle of the rat. Anat Rec. 1997, 248: 346-54.

Wang Y, Zhu S. Transfer of a branch of the anterior interosseous nerve to the motor branch of the median nerve and ulnar nerve. Chin Medl J (Engl). 1997, 110: 216-9. 